\title{
Aplicación de la Escala de disconfort del tracto vocal en detección, diagnóstico y terapéutica de la voz.
}

\author{
Application of the Argentinian version of the Vocal Tract \\ Discomfort Scale in the screening, diagnosis) and the \\ voice therapy.
}

\author{
María Alejandra Santi ${ }^{1}$, Andrea K. Romanoํ․ Silvina Montenegro ${ }^{23}$. \\ ${ }^{1}$ Escuela de Fonoaudiología. Facultad de Ciencias Médicas. Universidad Nacional de Rosario. Rosario; Argentina. \\ 2 Área de Metodología de la Investigación. Facultad Ciencias Médicas. Universidad Nacional de \\ Rosario. Rosario; Argentina. \\ ${ }^{3}$ Consejo de Investigación. Universidad Nacional de Rosario. Rosario; Argentina.
}

Autor por correspondencia: María Alejandra Santi — malejandrasanti@gmail.com

\section{Resumen}

Se describen las diferentes aplicaciones que puede tener la versión argentina de la escala Vocal Tract Discomfort (VTD), herramienta de autovaloración de síntomas o sensaciones que pueden presentarse en sujetos con alteraciones de la voz o en riesgo de presentarlas. Esta herramienta ha sido adaptada de su versión original en inglés y validada al español argentino para su utilización en detección de sujetos en riesgo vocal, para complementar el diagnóstico subjetivo y objetivo de la voz, para monitorear las sensaciones del paciente en la terapéutica y para poder comparar y discutir con resultados de investigaciones en diferentes países del mundo; donde también ha sido validada para su uso clínico e investigativo.

Palabras clave: Voz. Autovaloración. Escala de disconfort del tracto vocal.

\begin{abstract}
The different applications of the Argentinian version of the Vocal Tract Discomfort scale (VTD), a tool for self-assessment of symptoms or sensations that may occur in subjects with voice disorders or at risk of presenting them, are described. This tool has been adapted from its original English version and validated in Argentinean Spanish for its use in the detection of subjects at vocal risk, to complement the subjective and objective diagnosis of the voice, to monitor the patient's sensations in therapeutics and to discuss and compare and with the results of research in different countries around the world, where it has also been validated for clinical and research use.
\end{abstract}

Keywords: Voice. Self Assessment. Vocal Tract Discomfort Scale. 


\section{Introducción}

En el complejo proceso de valorar el estado de salud o enfermedad, el profesional pone a prueba su formación y experiencia. La práctica clínica individual no exige necesariamente el uso de escalas o cuestionarios, a diferencia de la investigación. En este caso, en algunas oportunidades el investigador se ve abocado a un reduccionismo ineludible, al necesitar comprimir conceptos anatómicos, fisiológicos, nosológicos, psicológicos, etc.; en variables que puedan ser medidas intentado suprimir el factor subjetivo del evaluado y del evaluador. En algunos casos es conveniente realizar una nueva escala, pero en otros será propicio utilizar una ya existente (1).

Para utilizar con garantía un cuestionario, es necesario comprobar que mide apropiadamente y de modo constante en las mismas circunstancias, que es sensible a los cambios de la situación clínica, que en la práctica no presenta dificultades de aplicación, etc. Toda la comprobación de sus características es lo que se llama proceso de validación. Se realiza inicialmente en el idioma original y debe reiterarse cuando se altera la estructura o cuando cambia el idioma en el que se aplica (2).

La mayoría de los cuestionarios se desarrollan en países de habla inglesa, pero aún para su uso en otros países con el mismo idioma, se debe considerar a qué poblaciones se aplicará, si las mismas provienen quizás de diferentes corrientes inmigratorias, etc., para comprobar su validez y evitar sesgos sistemáticos al aplicarla en un estudio. La adaptación transcultural de un cuestionario para su uso en un nuevo país, cultura, y/o idioma requiere que se establezca su fiabilidad y validez para alcanzar la equivalencia entre la versión original y la nueva. Cuando se pretende que las dos versiones de una escala en idiomas distintos sean comparables, hay que aportar pruebas de la comparabilidad de los instrumentos (3).

En la clínica fonoaudiológica es importante que la valoración de la voz sea multidimensional, acompañando a la evaluación perceptual y acústica del fonoaudiólogo, con protocolos de autovaloración de la calidad vocal (4). En esta perspectiva holística, es imprescindible contemplar la percepción del paciente sobre su calidad vocal, su disconfort, y el impacto de éstos en su calidad de vida. Las escalas de autovaloración suprimen presuntamente el valor subjetivo del evaluador, y ésta es la base de su utilidad en la investigación clínica.

Los protocolos de autovaloración o autoevaluación permiten medir el impacto de la disfonía en el paciente. Estas escalas otorgan relevancia a las sensaciones subjetivas que refieren los pacientes, de importancia tanto para el planteamiento de objetivos terapéuticos como para la organización de la terapia vocal.

Para que los pacientes puedan valorar su calidad de vida vocal, existen variados protocolos, que permiten cuantificar su sintomatología y medir cuánto le limita e interfiere su alteración en la voz. Elegir un protocolo con sus propiedades psicométricas demostradas, nos permite reducir la gran cantidad de terminología utilizada, simplificar, y dar reproducibilidad a la investigación.

Profesionales de la voz con mal uso y/o abuso vocal y pacientes con disfonías pueden experimentar además de los cambios en la calidad de la voz, molestias en el tracto vocal. El término disconfort, describe una experiencia subjetiva que representa una condición que compromete la funcionalidad ideal de una estructura, en este caso, del tracto vocal. Al ser estos síntomas o sensaciones experiencias subjetivas, son difíciles de evaluar, en consecuencia, Mathieson y colaboradores (5) desarrollaron la escala Vocal Tract Discomfort (VTD).

La escala VTD ha sido traducida al polaco (6), al portugués de Brasil (7), al alemán (8), al italiano (9), al árabe (10), al coreano (11), al persa (12) y se encuentra en proceso de adaptación al noruego. Al utilizarse instrumentos mundialmente aceptados, se abre la posibilidad de efectuar estudios entre diferentes países o culturas.

La escala VTD se ha validado para su uso en diversos países, por la gran utilidad de este protocolo de autovaloración en el proceso de diagnóstico y monitoreo de la terapéutica, sobre todo en disfonías músculo-tensionales $(6,9,10,12$ y 13). La escala no ha permitido diferenciar entre disfonías funcionales u orgánicas (6, 9 y 13), pero si en todas las validaciones se ha coincidido en la utilidad para evidenciar cambios pre-post terapia.

Para poder utilizarla en Argentina, se adaptó la VTD al español argentino (14) y se demostró la equivalencia cultural y lingüística de esta versión, llamada Escala de disconfort del tracto vocal (EDTV). Posteriormente, se realizó su validación (15). Evaluar la preservación de las propiedades psicométricas de la versión original de la EDTV permitió valorar la fiabilidad y validez de la misma. La Figura 1 muestra la escala original (VTD) y la Figura 2 su versión argentina (EDTV). 


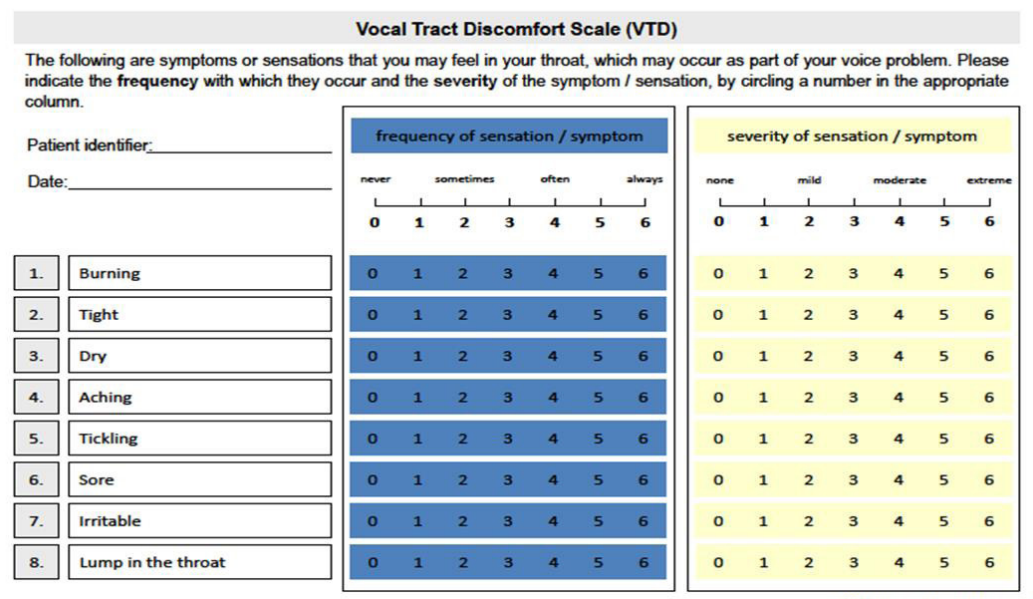

Figura 1: Versión original “Vocal Tract Discomfort-VTD”, a partir de la cual se realizó la adaptación y validación local.

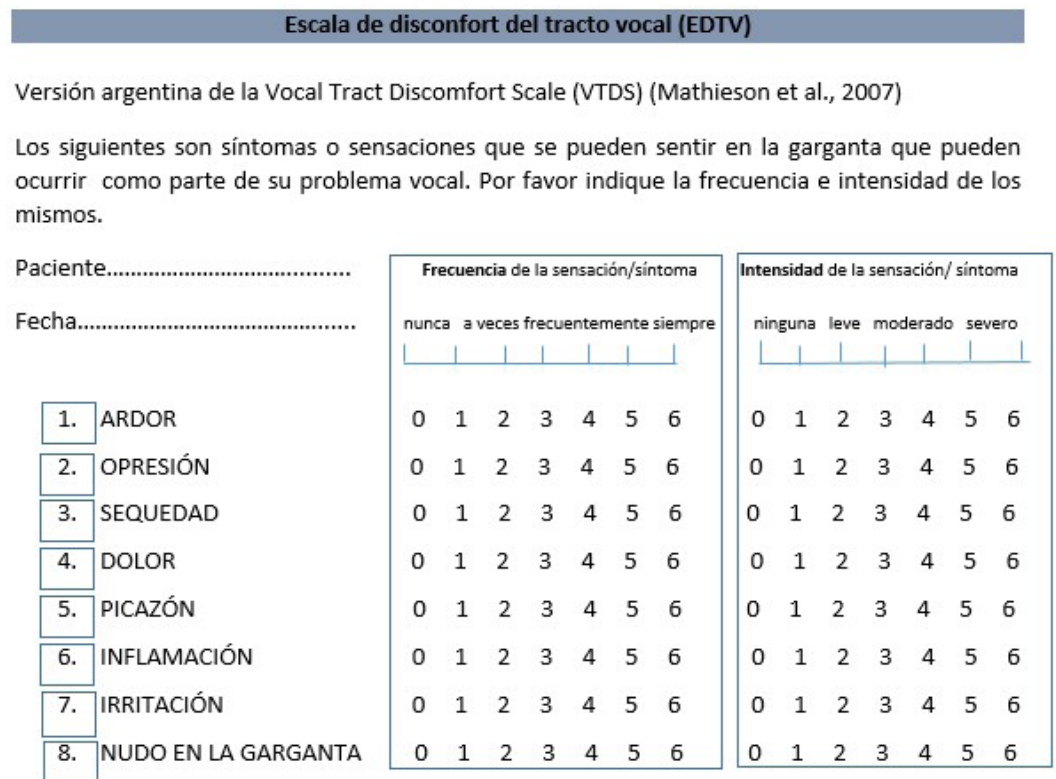

(Santi et al., 2018)

Figura 2: Escala de disconfort del tracto vocal (EDTV). Posteriormente, se realizó su validación (15). Evaluar la preservación de las propiedades psicométricas de la versión original de la EDTV permitió valorar la fiabilidad y validez de la misma.

La EDTV es un instrumento de evaluación altamente reproducible, fiable y valioso para evaluar a los pacientes argentinos con alteraciones de la voz. Es una herramienta útil para cuantificar la autopercepción del disconfort en el tracto vocal, y para la detección y el monitoreo de la sintomatología córporo-vocal. Asimismo, permite al paciente la toma de conciencia, en relación con las sensaciones o síntomas en el tracto vocal, registrando las mejorías y el alivio del disconfort, fortaleciendo así la adherencia terapéutica (14).

La EDTV evalúa ocho síntomas: ardor, opresión, sequedad, dolor, picazón, inflamación, irritación y sensación de nudo en la garganta. Se puntúan dos subescalas: frecuencia e intensidad, también puede calcularse el puntaje total sumando ambas. El sujeto marca sobre una escala de 0 a 6 la frecuencia del síntoma (de nunca a siempre) e indica la intensidad o severidad de los mismos (de ninguna a severa). En frecuencia el 0 es nunca, 1-2 a veces, 3-4 frecuentemente y 5-6 casi siempre y siempre. Con relación a la intensidad o severidad de la sintomatología 0 es ausencia del síntoma, 1-2 leve, $3-4$ moderado y 5-6 severo. Los resultados reflejan la percepción de la molestia del sujeto o paciente (15).

La subescala intensidad o severidad, puede servir para comparar cambios pre-post terapéutica permitiendo medir la efectividad de la misma. 


\section{Objetivo}

El objetivo de este artículo es revisar las diferentes aplicaciones de la Escala de disconfort del tracto vocal (EDTV), escala validada por Santi y colaboradores (15).

\section{Aplicación de la EDTV}

La EDTV puede ser utilizada para prevenir y detectar patologías vocales. En la campaña de prevención de alteraciones de la fonación realizada en conmemoración al Día Mundial de la Voz 2018, se aplicó en aproximadamente 1000 estudiantes de los Institutos de Profesorados de la ciudad de Rosario, considerados población de riesgo. El objetivo de esta autovaloración fue promover la reflexión sobre la presencia o ausencia de disconfort en el tracto vocal. Luego se les brindó pautas de higiene y técnica vocal para optimizar la fonación de los participantes.

En clínica e investigación, la EDTV puede utilizarse para comparar grupos sin y con patología vocal. Santi y col (14) aplicaron la misma a 30 sujetos sin patología vocal y 31 con disfonía músculotensional tipo I (DMT I: sin alteración en la mucosa laríngea). Los resultados más relevantes fueron que los sujetos con DMTI presentaron mayor cantidad de síntomas y más intensos que los sujetos sanos. Los síntomas más frecuentes en los sujetos con patología vocal fueron opresión, sequedad y nudo en la garganta (14).

Además, permite diferenciar el impacto de la disfonía en la calidad de vida vocal en distintas profesiones u ocupaciones. Koufmann e Isaacson (16) clasifican dicho impacto en 4 niveles, a saber: nivel 1 incluye la elite vocal, como cantantes y actores que se verían seriamente afectados. Nivel 2 , los profesionales de la voz hablada, como docentes que se afectarían moderadamente. Nivel 3, las voces ocupacionales, por ejemplo médicos o vendedores, a quienes solo les obstaculizaría su trabajo si sufrieran una alteración severa de la voz. Nivel 4, la voz no profesional, por ejemplo diseñadores o programadores de computación que casi no utilizan la voz para trabajar. Por último, se puede agregar un nivel 5: desempleados, estudiantes, amas de casa y jubilados como sugieren Gasparini y Behlau (17).

Santi y colaboradores compararon sujetos con DMT I y II (DMT II: con cambios estructurales en la mucosa laríngea), a partir de un muestreo por conveniencia, no detectando diferencias significativas en frecuencia e intensidad entre las diferentes profesiones u ocupaciones (15).

Al aplicar la EDTV en telemarketers (nivel 2), Martínez (18) concluyó que el síntoma con mayor frecuencia e intensidad fue sequedad. Similares resultados se obtuvieron en una investigación con adultos mayores con presbifonías (nivel 5) (19), donde los síntomas con valores superiores tanto para frecuencia como intensidad fueron: picazón, sequedad e irritación.

Otra aplicación de la escala es para estudiar la evolución del disconfort. Ávila y Lucci (20) suministraron la EDTV a docentes de primaria (nivel 2) en tres momentos del ciclo lectivo, permitiendo el seguimiento de la sintomatología a largo plazo. Este estudio visualizó el incremento de la cantidad de síntomas así como de la frecuencia e intensidad de los mismos a lo largo del año. La carga horaria incidió en la presentación de sintomatología, no así la antigüedad laboral. Los docentes con doble turno manifestaron presentar un número mayor de síntomas; siendo los más frecuentes y de mayor intensidad sequedad, picazón y ardor.

Por último, se aplicó la EDTV para medir cambios pre-post terapéutica. Santi y colaboradores (21) utilizaron la escala en 30 pacientes con DMT previa y posteriormente a recibir terapia manual laríngea de Mathieson. Se evidenciaron efectos positivos, al comprobarse la disminución de la intensidad de los síntomas, en todos los sujetos post terapia. Asimismo se constató desaparición de los síntomas en el $50 \%$ de los casos.

\section{Consideraciones finales}

A lo largo del recorrido propuesto, se ha podido observar la aplicación de la escala en diferentes situaciones. La enumeración de las mismas tiene como meta facilitarle a los fonoaudiólogos una herramienta interesante para la evaluación de la calidad vocal y alentarlos para su uso en la clínica y en el desarrollo de trabajos de investigación dónde se evalué su utilización en diferentes poblaciones y situaciones.

Actualmente se está desarrollando un proyecto de investigación de la Facultad de Ciencias Médicas, en el cual se aplica la EDTV, para medir el disconfort en profesionales de la salud usuarios de protección facial por COVID-19, estos resultados serán compartidos en próximas publicaciones. 
Sería interesanteutilizar laEDTV, para objetivar cambios pre-post varias sesiones de fonoaudiología como se realizó con la versión persa de la VTD (12).

Recientemente, se ha incorporado la EDTV a la aplicación Web Clinical Voice box (22), herramienta para la evaluación de la voz, que está disponible en forma gratuita para fonoaudiólogos y otorrinolaringólogos. Cabe destacar que como afirman Behlau y colaboradores (23) ninguna de las evaluaciones médicas ni fonoaudiológicas, utilizadas en forma aislada, resulta ser una herramienta diagnóstica perfecta. Por lo tanto, es necesario que en la valoración de la calidad vocal se contemplen los parámetros vocales perceptuales, acústicos y la sintomatología de cada sujeto en su completud y complejidad; correlacionándolos con su historia clínica general y vocal.

Fuentes de financiamiento: no presenta.

\section{Referencias bibliográficas}

1. Carvajal A, Centeno C, Watson R, Martínez M, Sanz Rubiales Á. ¿Cómo validar un instrumento de medida de la salud?. Anales Sis San Navarra. 2011; 34(1): 63-72.

2. Luján Tangarife JA, Cardona Arias JA. Construcción y validación de escalas de medición en salud: revisión de propiedades psicométricas. Archivos de Medicina. 2015; 11 (3). doi: 10.3823/1251

3. Beaton DE, Bombardier C, Guillemin F, Bosi Ferraz M. Guidelines for the Process of Cross-Cultural Adaptation of Self-Report Measures. Spine. 2000; 25 (24): 3186-3191.

4. Dejonckere PH, Bradley P, Clemente P, et al. A basic protocol for functional assessment of voice pathology, especially for investigating the efficacy of (phonosurgical) treatments and evaluating new assessment techniques. Guideline elaborated by the Committee on Phoniatrics of the European Laryngological Society (ELS). Eur Arch Otorhinolaryngol. 2001; 258 (2): 77-82.

5. Mathieson L, Hirani SP, Epstein R, Baken J, Wood G, Rubin JS. Laryngeal manual therapy: A preliminary study to examine its effects in the management of muscle tension dysphonia. J Voice. 2009; 23(3): 353-366.

6. Woznicka E, Niebudek-Bogusz E, Kwiecień J, Wiktorowicz J, Sliwińska-Kowalska M. Applicability of the vocal tract discomfort (VTD) scale in evaluating the effects of voice therapy of occupational voice disorders. Medycyna Pracy. 2012; 63(2): 141-152.

7. Rodrigues G, Zambon F, Mathieson L, Behlau M. Vocal Tract Discomfort in Teachers: Its Relationship to Self-Reported Voice Disorders. J Voice. 2013; 27 (4): 473-480.

8. Lukasky J, Brockmann-Bauser M, Beushausen U. Transcultural Adaptation and Validation of the German Version of the Vocal Tract Discomfort Scale. J Voice. 2017; 31(2): 261 el-e8.

9. Robotti C, Mozzanica F, Pozzali I, et al. Cross- cultural Adaptation and Validation of the Italian Version of the vocal tract Discomfort Scale (I-VTD). J Voice. 2019; 33 (1): 115-123.

10. Darawsheh W B, Shdaifat A, Natour YS. Validation of the Arabic version of vocal tract discomfort scale. Logopedics Phoniatrics Vocology. 2019; 45 (2): 82-90.

11. Lee HN, Yoo JY, Han JH, Park YS, Jung DY, Park JH. Transcultural Adaptation and Validation of the Korean Version of the Vocal Tract Discomfort Scale. J Voice. 2020; S0892-1997(20)30158-2. doi: 10.1016/j.jvoice.2020.04.030. PMID: 32576525.

12. Torabi H, Khoddami S, Ansari NN y Dabirmoghaddam P. The Vocal Tract Discomfort Scale validity and reliabibility of the Persian in the assessment of patients with muscle tension dysphonia. J Voice. 2016 ; 30 (6): 711-716.

13. Kristiane M, Lierde V, Bodt M, Dhaeseleer E, Wuyts F, Claeys S. The treatment of muscle tension dysphonia: A comparison of two treatment techniques by means of an objective multiparameter approach. J Voice. 2010; 24 (3): 294-301.

14. Santi MA, Romano A K, Montenegro S. Adaptación de la Vocal Tract discomfort Scale al español argentino. Revista Facultad de Ciencias Médicas. 2018; 27 (3): 34-39.

15. Santi MA, Romano AK, Dajer ME, Montenegro S, Mathieson L. Vocal Tract Discomfort Scale: Validation of the Argentine Version. J Voice. 2018; 34 (1): 158.el-158.e70892-199.

16. Koufman JA, Isaacson G. The spectrum of vocal dysfunction. Otolaryngol Clin North Am. 1991; 24(5): 985-88

17. Gasparini G, Behlau M. Quality of life: validation of the Brazilian version of the voice-related quality-of-life(V-RQOL) measure. J Voice. $2007 ; 23$ (1): 76-81.

18. Martinez C. Estudio descriptivo: escala de disconfort del tracto vocal y cualidades de la voz en telemarketer del call center de la Facultad de Ciencias Médicas de la Universidad Nacional de Rosario. (Tesis de licenciatura). Escuela de Fonoaudiología. Facultad de Ciencias Médicas Universidad Nacional de Rosario. 2018.

19. Camposaragna O, Leguizamón I. Autoevaluación de los síntomas de disconfort del tracto vocal en adultos mayores. (Tesis de licenciatura). Escuela de Fonoaudiología. Facultad de Ciencias Médicas, Universidad Nacional de Rosario. 2019.

20. Ávila AN, Lucci MA. Estudio prospectivo: síntomas de disconfort del tracto vocal en docentes de nivel primario de la ciudad de Pérez en el año lectivo 2018. (Tesis de licenciatura). Escuela de Fonoaudiología. Facultad de Ciencias Médicas, Universidad Nacional de Rosario. 2018.

21. Santi MA, Romano AK, Montenegro S, Gurlekian JA. Técnica de masaje laríngeo: validación acústica mediante índices integrados. Revista de Investigación en Logopedia. 2017; 7 (1),: 71- 88.

22. Jeréz M. Clinical Voice box. Disponible en: https://clinicalvoicebox.com/ o https://www.afonoaudiologicas.com/. [Consultado:4 de marzo 2021]

22. Behlau M, Zambón F, Moreti F, Oliveira G, de Barros Couto E Jr. Voice Self-assessment Protocols: Different Trends Among Organic and Behavioral Dysphonias. J voice. 2017;31(1):. 112.e13-112.e27. 